\title{
A least-square-driven functional networks type-2 fuzzy logic hybrid model for efficient petroleum reservoir properties prediction
}

\author{
Fatai Anifowose · Jane Labadin • Abdulazeez Abdulraheem
}

Received: 31 January 2012/ Accepted: 7 December 2012/Published online: 18 January 2013

(C) Springer-Verlag London 2013

\begin{abstract}
Various computational intelligence techniques have been used in the prediction of petroleum reservoir properties. However, each of them has its limitations depending on different conditions such as data size and dimensionality. Hybrid computational intelligence has been introduced as a new paradigm to complement the weaknesses of one technique with the strengths of another or others. This paper presents a computational intelligence hybrid model to overcome some of the limitations of the standalone type-2 fuzzy logic system (T2FLS) model by using a least-square-fitting-based model selection algorithm to reduce the dimensionality of the input data while selecting the best variables. This novel feature selection procedure resulted in the improvement of the performance of T2FLS whose complexity is usually increased and performance degraded with increased dimensionality of input data. The iterative least-square-fitting algorithm part of functional networks (FN) and T2FLS techniques were combined in a hybrid manner to predict the porosity and permeability of North American and Middle Eastern oil and gas reservoirs. Training and testing the T2FLS block of the hybrid model with the best and dimensionally reduced input variables caused the hybrid model to perform better with higher correlation coefficients, lower root mean square errors, and less execution times than the standalone T2FLS model. This work has demonstrated the promising
\end{abstract}

F. Anifowose $(\bowtie) \cdot$ J. Labadin

Faculty of Computer Science and Information Technology, Universiti Malaysia Sarawak, 94300 Kota Samarahan,

Sarawak, Malaysia

e-mail: fanifowose@gmail.com

A. Abdulraheem

Department of Petroleum Engineering, King Fahd University of Petroleum and Minerals, Dhahran 31261, Saudi Arabia capability of hybrid modelling and has given more insight into the possibility of more robust hybrid models with better functionality and capability indices.

Keywords Hybrid artificial intelligence $\cdot$ Functional networks · Type-2 fuzzy logic · Petroleum reservoir . Least-square-fitting algorithm

\section{Introduction}

Porosity and permeability are two important oil and gas reservoir properties which have to do with the amount of fluid contained in a reservoir and its ability to flow. These properties have significant impact on petroleum field operations and reservoir management [1]. They are usually measured in the laboratory on plugs extracted from the core of wells drilled for oil and gas exploration. The data are valuable for relating the two properties whose values are not directly measurable from well logs since they are purely natural phenomena. Both porosity and permeability data serve as standard indicators of reservoir quality in the oil and gas industry. This process, called petroleum reservoir characterization, is used for quantitatively describing various reservoir properties in spatial variability by using available field and laboratory data. The process plays a crucial role in modern reservoir management: making sound reservoir decisions and improving the reliability of the reservoir predictions. These properties make significant impacts on petroleum field operations and reservoir management [2].

Since the laboratory measurements are usually costly and time-consuming, artificial intelligence (AI) techniques have been successfully used in the prediction of these properties to an acceptable degree of accuracy [1,3-5]. However, each AI technique has certain limitations that would not make 
their use desirable in certain conditions such as in small dataset scenarios [6,7] and high dimensionality of data conditions $[8,9]$. Some AI techniques such as type-2 fuzzy logic are sensitive to the dimensionality of data $[9,10]$ such that even a little reduction in the number of features is expected to make a significant improvement in their performance indices. Hence, combining such techniques with others that have the capability of addressing some of the limitations in the former, such as reducing the data dimensionality, would be a welcomed development. Such hybridization tasks will improve the accuracy of predictions, which would in turn, increase the confidence in the results and hence improve the overall efficiency of oil and gas exploration and production activities.

The hybridization of two or more AI techniques, known as hybrid computational intelligence (HCI), to create a single integrated model, is becoming increasingly popular. The increased popularity lies in their extensive success in real-world complex problems such as in the characterization of oil and gas reservoirs [1,6], network intrusion detection [11], biometric face recognition [12], bioinformatics [13, 14], financial credit risk assessment [15], multimedia processing [16], and control systems [17].

In this work, the desirable qualities of LS-fitting algorithm of functional networks (FN) and type-2 fuzzy logic (T2FLS) were combined to predict two properties of oil and gas reservoirs, namely porosity and permeability, with better performance indicators. Our motivations for this work include the quest for higher performance accuracy in the prediction of oil and gas reservoir properties; the recently increasing popularity of hybrid intelligent systems; the reported success of these hybrid systems in many real-world complex problems; the need to complement the weaknesses of one algorithm with the strengths of the other and hence to combine the cooperative and competitive characteristics of the individual techniques; and the existing theoretical and experimental justifications that hybrids produce more accurate results than the individual techniques used separately $[2,6,18]$.

The considerable number of applications of hybrid techniques indicate, on one hand, the keen interest of researchers in this new concept and, on the other hand, the need for better components that will yield hybrid models that are simpler in architecture and more efficient in performance.

The rest of this paper is organized as follows: Sect. 2 presents some background knowledge on the petroleum reservoir characterization process, porosity, and permeability. Section 3 reviews some of the previous works related to this study. Section 4 describes the datasets, experimental methodology, and the criteria for the performance evaluation of this work. Section 5 presents the results with a detailed discussion. Conclusion is presented in Sect. 6 while our plan for further research is presented in Sect. 7.

\section{Background knowledge}

\subsection{Petroleum reservoir characterization}

Oil and gas reservoir characterization plays a crucial role in modern reservoir management. It helps to make sound reservoir decisions and improves the asset value of the oil and gas companies. It maximizes the integration of multidisciplinary data and knowledge, and hence improves the reliability of reservoir predictions. The ultimate goal is "a reservoir model with realistic tolerance for imprecision and uncertainty" [2]. Furthermore, it is the process between the discovery phase of a reservoir and its management phase. The process integrates the technical disciplines of petroleum reservoir engineering, geology, geophysics, oil and gas production engineering, petrophysics, economics, and data management. The key objectives of reservoir characterization focus on modelling each reservoir unit, predicting well behavior, understanding past reservoir performance, and forecasting future reservoir conditions.

The petroleum reservoir characterization process usually involves a well logging technique that makes measurements in oil and gas wells that have been drilled. Probes are designed and lowered in these wells to measure the physical and chemical properties of rocks as well as the fluids contained in them. Much information can be obtained from samples of rock brought to the surface in cores or bit cuttings, or from other clues while drilling, such as the rate of penetration, reservoir pressure, and bottom-hole temperature. However, the greatest amount of information comes from well logs which results from the probes. The resulting measurements, known as geophysical well logs, are recorded graphically or digitally as a function of depth.

Although the most commonly used logs are for the correlation of geological strata and location of hydrocarbon zones, there are many other important subsurface parameters that need to be detected or measured. Also, different borehole and formation conditions can require different tools to measure the same basic property [19]. Some of the numerous uses of logs in petroleum engineering include the identification of potential reservoir rocks and bed thickness, determination of porosity, estimation of permeability, location of and quantification of the amount of hydrocarbons, and estimation of water salinity.

\subsection{Porosity and permeability}

Porosity is the percentage of voids and open spaces in a rock or sedimentary deposit. The greater the porosity of a rock, the greater its ability to hold water and other materials, such as oil, will be. It is an important consideration when attempting to evaluate the potential volume of hydrocarbons contained in a reservoir. Sedimentary 